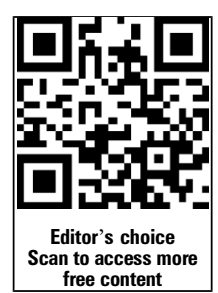

For numbered affiliations see end of article.

Correspondence to Dr Paul Reavley, Emergency Department, Bristol Royal Infirmary, Upper Maudlin Street, Bristol BS2 8HW, UK paulreavley@doctors.org.uk

Received 9 November 2013 Revised 17 October 2014 Accepted 10 November 2014 Published Online First 27 November 2014
CrossMark

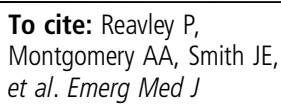

\title{
Randomised trial of the fascia iliaca block versus the '3-in-1' block for femoral neck fractures in the emergency department
}

\author{
Paul Reavley, ${ }_{1}^{1}$ Alan A Montgomery, ${ }^{2}$ Jason E Smith, ${ }^{3,4}$ Simon Binks, ${ }^{5}$ \\ Judith Edwards, ${ }^{1}$ Georgina Elder, ${ }^{1}$ Jonathan Benger ${ }^{1,6}$
}

\section{ABSTRACT}

Introduction Femoral neck fractures are a common

and painful injury. Femoral nerve blocks, and a variant of this technique termed the ' 3 -in-1' block, are often used in this patient group, but their effect is variable. The fascia iliaca compartment block (FIB) has been proposed as an alternative, but the relative effectiveness of the two techniques in the early stages of care is unknown. We therefore compared the FIB versus the 3in-1 block in a randomised trial conducted in two UK emergency departments.

Methods Parallel, two-group randomised equivalence trial. Consenting patients $>18$ years with a femoral neck fracture were randomly allocated to receive either a FIB or a 3 -in-1 block. The primary outcome was pain measured on a $100 \mathrm{~mm}$ visual analogue scale at $60 \mathrm{~min}$. The between-group difference was adjusted for centre, age, sex, fracture type, pre-block analgesia and pre-block pain score.

Results 178 patients were randomised and 162 included in the primary analysis. The mean $100 \mathrm{~mm}$ visual analogue pain scale score at 60 min was $38 \mathrm{~mm}$ in the FIB arm and $35 \mathrm{~mm}$ in the 3-in-1 arm. The adjusted difference between the arms was $3 \mathrm{~mm}$, with a $95 \% \mathrm{Cl}(-4.7$ to 10.8$)$ that excluded a clinically important difference between the two interventions. Conclusions FIB is equivalent to the 3 -in-1 block for immediate pain relief in adult neck of femur fractures. Trial registration number ISRCTN16152419.

\section{INTRODUCTION}

Around 75000 people suffer a hip fracture each year in the UK. ${ }^{1}$ These injuries are associated with considerable mortality and morbidity in a predominantly elderly patient population. ${ }^{1}$

Integral to the emergency department (ED) management of hip fracture is the rapid provision of effective analgesia. The UK College of Emergency Medicine standard is that $100 \%$ of patients in moderate or severe pain should be offered or receive analgesia within $60 \mathrm{~min}$ of arrival at ED. ${ }^{2}$ Peripheral nerve block techniques are considered in patients for whom paracetamol and opiates do not provide adequate analgesia, or to limit opioid dosage. ${ }^{1}$

There are two principal techniques described: the fascia iliaca compartment block (FIB) and the 3-in-1 block. FIB was first described by Sharrock ${ }^{3}$ in 1989. The 3-in-1 block was first described by Winnie et $a l^{4}$ in 1973 Both are single-injection anterior thigh approach techniques that aim to

\section{Key messages}

What is already known on this subject

- Both 3-in-1 block and fascia iliaca block (FIB) are effective techniques for relieving pain in femoral neck fractures, but there have been no large trials directly comparing the two techniques in the emergency department.

- Recent evidence show that nerve stimulator 3-in-1 blocks are superior to FIB for pain relief in femoral neck fractures, but most emergency departments now have access to ultrasound.

- This trial was performed to identify the most effective nerve block technique for relieving femoral neck fracture pain.

\section{What this study adds}

- Emergency medicine physicians can be confident that both 3-in-1 block and FIB provide pain relief in femoral neck fractures with no clinically significant difference between the two techniques in the emergency department setting.

- Blocks can be effectively delivered without ultrasound guidance, but future research should be undertaken to compare FIB with ultrasound-guided 3-in-1 blocks.

block the femoral, obturator and lateral femoral cutaneous nerves.

While each has been studied individually, there is very little evidence comparing the two techniques in the early stages of care, although one study showed superiority of a standard femoral nerve block over FIB in a nurse-delivered acute pain service. $^{5}$

With the majority of evidence suggesting similar effect, this study was designed to establish whether the fascia iliaca compartment block is equivalent to the 3 -in-1 femoral nerve block for immediate pain relief in adult neck of femur fractures.

\section{METHODS}

\section{Design, setting and participants}

We completed a two-group parallel randomised equivalence trial at University Hospitals Bristol NHS Foundation Trust and Plymouth Hospitals NHS Trust. Consenting adult patients (18 years and older) with a radiographically confirmed femoral 
neck fracture were invited to participate and offered a patient information leaflet by either an emergency medicine consultant or senior trainee. Following written consent, they were randomly allocated to receive either a FIB or 3-in-1 block. The exclusion criteria were patient refusal, an abbreviated minimental state examination of $7 / 10$ or less, other distracting painful pathology, contraindication to local anaesthetic agents, an inability to speak or understand spoken English and injury $>24$ h previously.

\section{Interventions}

FIBs were performed using anatomical landmarks. Bupivacaine $0.5 \%$ solution was injected at a point $1 \mathrm{~cm}$ perpendicular and distal to the junction of the middle and outer thirds of a line drawn between the anterior superior iliac crest and the pubic tubercle. The dose used was $2 \mathrm{mg}$ per kilogram up to a maximum of $150 \mathrm{mg}$ ( $30 \mathrm{~mL}$ of $0.5 \%$ bupivacaine). Where the dose was $<150 \mathrm{mg}$, the total volume of solution was diluted to $30 \mathrm{~mL}$ with $0.9 \%$ sodium chloride. To identify the correct compartment, an $18 \mathrm{~g}$ Tuohy needle was inserted at $90^{\circ}$ and advanced until two distinct 'pops' or loss of resistance were felt as first the fascia lata and then fascia iliaca were penetrated.

To deliver the 3-in-1 block, the femoral nerve was identified either anatomically by ultrasound or by nerve stimulation. This was a deviation from the original protocol of using only nerve stimulator guidance. Because of the prevalence and preference for ultrasound guidance and user-reported difficulties with nerve stimulator guidance, a pragmatic modification to the protocol allowing the 3 -in-1 block to be delivered by the technique that the operator was trained and current in was approved and initiated. The femoral nerve was identified anatomically using an injection point just lateral to the femoral arterial pulse at the femoral crease. If using a nerve stimulator, proximity was confirmed with a linear patella jerk at $30 \mathrm{mV}$. If using ultrasound, the block was delivered under continuous visualisation. Once located $2 \mathrm{mg}$ per kilogram of $0.5 \%$ bupivacaine was injected with distal pressure over the femoral nerve during and for $30 \mathrm{~s}$ after the injection. No head-down tilt was applied to the patient. Again, where the dose was $<150 \mathrm{mg}(30 \mathrm{~mL} 0.5 \%$ bupivacaine) the volume injected was diluted to $30 \mathrm{~mL}$ with $0.9 \%$ sodium chloride.

\section{Randomisation}

Participants were allocated on a 1:1 ratio using a secure online computer-generated randomisation service provided by the Bristol Randomised Trials Collaboration, a UK Clinical Research Collaboration-registered clinical trials unit. This concealed allocation from recruiting staff. Allocation was stratified by centre, and minimised, retaining a random element, by age, sex, fracture type, pre-block analgesia and pre-block pain score. ${ }^{6}$

\section{Outcomes and blinding}

The primary outcome was pain measured using a $100 \mathrm{~mm}$ visual analogue scale (VAS) at $60 \mathrm{~min}$ after the block was delivered. Secondary outcome measures were pain at $30 \mathrm{~min}$ after block delivery, analgesia consumption in the preoperative period up to $24 \mathrm{~h}$ postadmission, analysed separately by drug type, and length of hospital stay. The VAS scores were recorded by the patient and collected by a member of the ED nursing staff. Patients were asked to record their pain by placing a mark on a $100 \mathrm{~mm}$ non-graduated line, representing no pain at all to the worst pain ever at $100 \mathrm{~mm}$. Patients were unlikely to recognise the type of block allocated and were therefore blinded to treatment allocation. The member of ED staff collecting data booklet was not blinded. Analgesia consumption was recorded from the patient's drug prescription chart by a research nurse and research assistant who were not blinded.

\section{Sample size}

The study was powered to investigate equivalence between the FIB and 3-in-1 blocks in terms of patient-reported $0-100 \mathrm{~mm}$ VAS pain score. Previous studies report an SD of 21 points on a $0-100 \mathrm{~mm}$ VAS and a difference of 13 points as the minimum clinically important difference. ${ }^{7}$ With a conservative difference beyond which the groups would not be regarded as equivalent of $10 \mathrm{~mm}, 80 \%$ power and $2.5 \%$ two-sided $\alpha$, the study required 86 patients per arm. Allowing for up to $10 \%$ noncollection of the primary outcome, we aimed to recruit 190 participants.

\section{Statistical methods}

We used appropriate descriptive statistics to examine balance between the trial arms in baseline characteristics. Analyses of primary and secondary outcomes were conducted on an intention-to-treat basis without imputation-that is, all patients were analysed according to their randomised groups, except for those who did not provide follow-up data. We used multivariable linear regression models to investigate between-group differences, adjusted for centre, age, sex, fracture type, pre-block analgesia and pre-block pain score as stratification and minimisation variables.

In prespecified subgroup analyses using interaction terms in the primary regression model, we explored whether any differences between the trial arms differed according to fracture type and pre-block analgesia type. Additional subgroup analyses for the primary outcome were conducted by gender and baseline pain score. These analyses were not prespecified in the protocol. However, in discussions prior to conducting the data analysis, we defined these to be of interest but are to be considered exploratory.

Following the protocol change, we explored the primary outcome for participants who had the 3-in-1 block delivery guided by ultrasound, nerve stimulator and anatomically. We conducted a sensitivity analysis by excluding all participants with nerve stimulator guidance in the 3 -in-1 arm, and all participants in the FIB arm randomised up to and including the same date as the final nerve stimulator-guided 3-in-1 block.

Statistical analyses were conducted using Stata V.11.

\section{RESULTS}

\section{Participant flow}

We recruited and randomised patients between December 2008 and December 2012. A total of 178 participants were randomised, with 162 included in the primary analysis. Figure 1 illustrates the flow of patients during the trial, including reasons why primary outcome data were not available for 16 participants. The trial arms were well balanced at baseline (table 1). Primary outcome data were obtained from $91 \%$ of participants. At the Plymouth site, the routine technique for the 3-in-1 block was anatomical, and at Bristol it was ultrasound.

\section{Primary and secondary outcomes}

The adjusted difference in mean pain scores at $60 \mathrm{~min}$ comparing 3-in-1 with FIB was $3.0 \mathrm{~mm}$, with $95 \% \mathrm{CI}-4.7 \mathrm{~mm}$ to $10.8 \mathrm{~mm}$ (table 2).

Pain scores after $30 \mathrm{~min}$ were equivalent between the arms (table 2). There was evidence that length of stay was longer in 
Figure 1 Participant flow diagram.

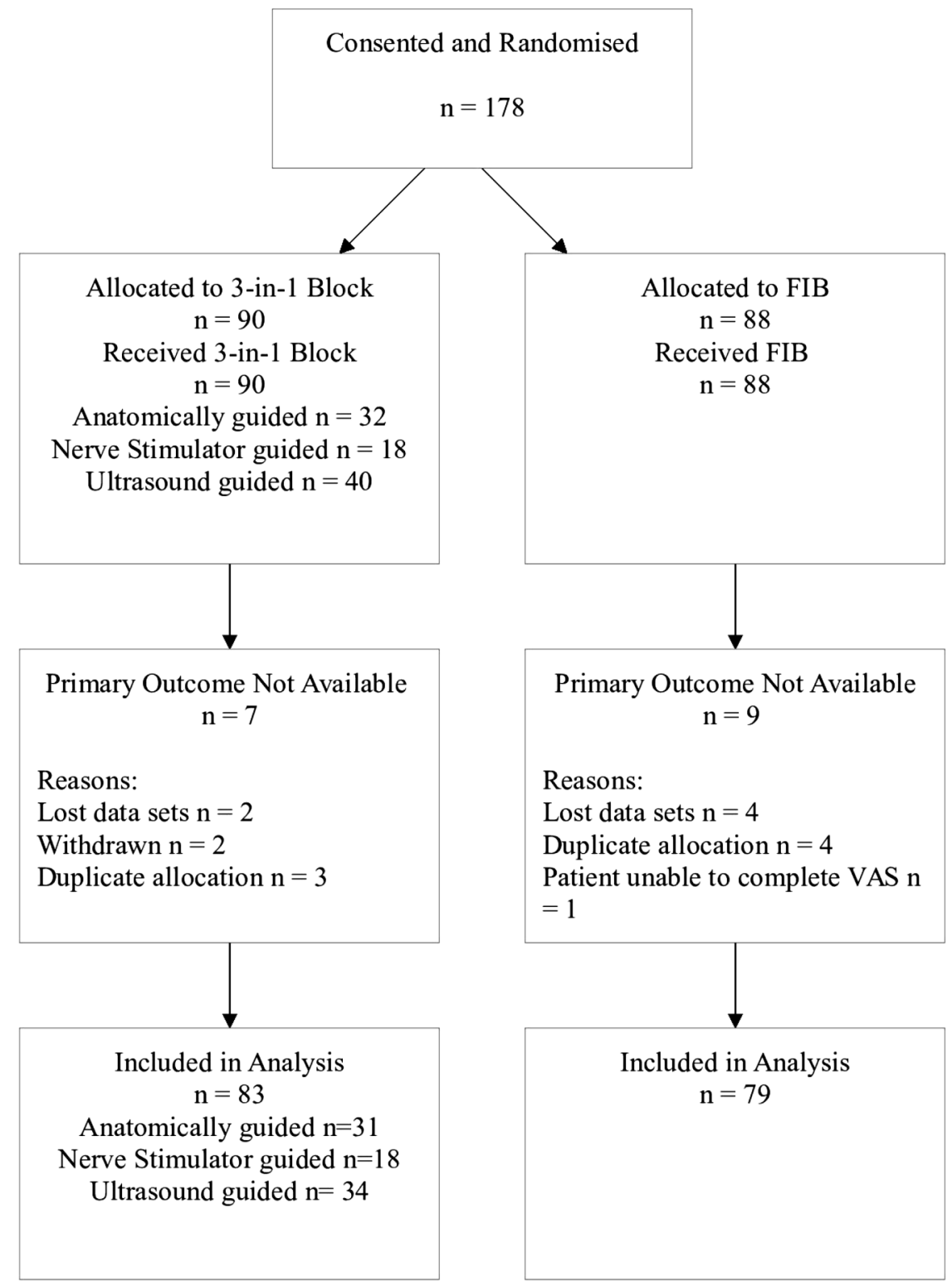

Table 1 Baseline characteristics of randomised participants

\begin{tabular}{lcc}
\hline & 3-in-1 ( $\mathbf{n = 9 0 )}$ & FIB ( $\mathbf{n = 8 8})$ \\
\hline Age in years, mean (SD) & $78(11)$ & $80(10)$ \\
Women, $\mathrm{n}(\%)$ & $65(72)$ & $66(75)$ \\
Fracture type, $\mathrm{n}(\%)$ & & \\
$\quad$ Subcapital & $43(48)$ & $42(48)$ \\
$\quad$ Intertrochanteric & $37(41)$ & $34(38)$ \\
$\quad$ Basicervical & $10(11)$ & $12(14)$ \\
Centre, $\mathrm{n}(\%)$ & & \\
$\quad$ Bristol & $57(51)$ & $55(49)$ \\
$\quad$ Plymouth & $33(50)$ & $33(50)$ \\
Pre-block analgesia $\mathrm{n}(\%)$ & & $10(11)$ \\
$\quad$ None & $5(6)$ & $8(9)$ \\
$\quad$ Oral only & $11(12)$ & $8(9)$ \\
$\quad$ Intravenous paracetamol & $8(9)$ & $62(71)$ \\
$\quad$ Intravenous morphine & $66(73)$ & $65(26)$ \\
Pre-block pain score, mean (SD) & $64(26)$ & \\
\hline FIB, fascia iliaca block. & &
\end{tabular}

the FIB group compared with the 3-in-1 group (95\% CI for ratio of geometric mean 1.07 to 1.57 ) (table 2).

Table 3 shows that the use of analgesia after randomisation and prior to operation was very similar between the two groups. Oral paracetamol and oral codeine were the most commonly used analgesics. Comparing means for FIB versus 3-in-1 yielded $95 \%$ CIs of -305 to $275 \mathrm{mg}$ for oral paracetamol and -17 to $52 \mathrm{mg}$ for oral codeine, respectively. Between-group comparisons for the other drugs were not performed due to small numbers.

There was no evidence of any interaction between treatment arm and fracture type $(\mathrm{p}=0.82)$, pre-block analgesia $(\mathrm{p}=0.11)$, gender $(p=0.93)$ and baseline pain score $(p=0.16)$ for the primary outcome (data not shown).

Mean (95\% CI) VAS pain scores at 60 min for patients in the 3-in-1 arm who had block delivery guided by nerve stimulator, anatomical and ultrasound were $32 \mathrm{~mm}(22-43), 35 \mathrm{~mm}(26-43)$ and $37 \mathrm{~mm}$ (28-47), respectively. Excluding primary outcome data for those with nerve stimulator-guided 3-in-1 blocks $(n=18)$ and FIB delivered up to the date of protocol change $(n=14)$, the 
Table 2 Pain score and length of stay

\begin{tabular}{|c|c|c|c|c|c|c|c|}
\hline & \multicolumn{2}{|l|}{ FIB } & \multicolumn{5}{|l|}{ 3-in-1 } \\
\hline & Mean (SD) & $\mathbf{N}$ & Mean (SD) & $\mathrm{N}$ & Difference & $95 \% \mathrm{Cl}$ & p Value \\
\hline \multicolumn{8}{|l|}{ Primary outcome } \\
\hline Pain score at $60 \mathrm{~min}$ in $\mathrm{mm}$ & $38(25)$ & 79 & $35(25)$ & 83 & 3.0 & -4.7 to 10.8 & 0.44 \\
\hline \multicolumn{8}{|l|}{ Secondary outcomes } \\
\hline Pain score at $30 \mathrm{~min}$ in $\mathrm{mm}$ & $44(26)$ & 80 & $45(24)$ & 85 & -0.7 & -8.1 to 6.6 & 0.85 \\
\hline Length of stay in days & $13.5^{*}$ (9.5 to 28.5$)$ & 84 & $10 *(7$ to 16$)$ & 87 & $0.26 t$ & 0.07 to 0.45 & 0.006 \\
\hline
\end{tabular}

difference in mean VAS score at $60 \mathrm{~min}$ was $5 \mathrm{~mm}(95 \% \mathrm{CI}-4$ to 13$)$.

\section{DISCUSSION}

This trial has shown that the FIB and 3 -in-1 blocks are equivalent in reducing pain scores at $60 \mathrm{~min}$ using a $0-100 \mathrm{~mm}$ VAS. While the upper $95 \%$ confidence limit of the difference measured marginally exceeded the conservative $10 \mathrm{~mm}$ equivalence limit proposed at trial design, it is still well within the $13 \mathrm{~mm}$ difference considered previously as minimally clinically important. ${ }^{7}$ Subgroup analysis according to fracture type suggested that the blocks are equally effective in intracapsular and extracapsular fracture types. Similarly, there was no evidence that the blocks were differentially effective according to gender, age or pre-block analgesia. There was an increase in length of stay in the FIB arm. This is difficult to explain, though we think it is unlikely to be due to the block itself, but related to patient and service factors not considered in this study.

\section{Limitations}

This trial was a pragmatic and realistic representation of nerve block practice for femoral neck fractures in EDs today where $66 \%$ of nerve blocks are delivered anatomically, and the rest nerve stimulator or ultrasound guided. ${ }^{8}$ The protocol change to allow the 3 -in-1 blocks to be given using the technique in which the operator was trained may be considered a limitation. Newman et $a l^{5}$ showed that a nerve stimulator-guided 3-in-1 block was superior to FIB. In their study, 93\% of the blocks were delivered by two operators and the difference between

Table 3 Analgesia consumption post-block in preoperative phase up to $24 \mathrm{~h}$

\begin{tabular}{|c|c|c|c|c|}
\hline & \multicolumn{4}{|l|}{ Sample size* } \\
\hline & \multicolumn{2}{|l|}{ FIB } & \multicolumn{2}{|l|}{3 in 1} \\
\hline & $\begin{array}{l}\text { Median } \\
\text { (10th-90th } \\
\text { centiles) }\end{array}$ & $\mathrm{N}$ & $\begin{array}{l}\text { Median } \\
\text { (10th-90th } \\
\text { centiles) }\end{array}$ & $\mathbf{N}$ \\
\hline Intravenous paracetamol (mg) & $1000(1000-4000)$ & 5 & $1000(1000-1000)$ & 12 \\
\hline Oral paracetamol (mg) & $3000(2000-4000)$ & 75 & $3000(2000-4000)$ & 74 \\
\hline Oral codeine (mg) & $180(32-240)$ & 31 & $120(60-240)$ & 35 \\
\hline Intravenous morphine (mg) & $5(3-20)$ & 7 & $8(5-36)$ & 6 \\
\hline Oral ibuprofen (mg) & $600(400-800)$ & 2 & 800 (NA) & 1 \\
\hline
\end{tabular}

the mean VAS score reduction was $0.9 \mathrm{~cm} \quad(95 \%$ CI 0 to $1.8 \mathrm{~cm}, \mathrm{p}=0.047$ ), which would not be considered as clinically important. ${ }^{7}$ There were no restrictions on use of pre-block analgesia in our study, which could also be viewed as a limitation. However, allocation was minimised by pre-block analgesia, resulting in no important difference in pre-block analgesia between the two groups. The number of participants included in the primary analysis was 162 compared with the target of 172, which may have resulted in reduced precision of between-group estimated differences in outcome. The primary outcome was self-reported by participants. It is unlikely that they would have been aware of which block they had received, therefore this outcome is at low risk of bias due to unblinding. However, staff collecting the self-completed data booklets from the patients were not blinded, and this could have introduced a source of bias.

Analgesia consumption and length of stay were extracted from patient medical records by unblinded research staff. However, as these are objective outcomes, we again consider the risk of bias to be low.

Aside from diagnosis, management of acute comorbidities and preparation for surgery, pain relief is vital for patients with a fractured neck of femur and is a nationally audited quality benchmark for EDs. Patients are often frail and elderly, and injury is often a result of multiple physical and cognitive factors. Safe but effective analgesia can be a challenge, and usually involves the administration of parenteral opiates. While opiates can exacerbate or cause delirium, evidence also shows that cognitively intact patients with inadequately treated pain from hip fracture are nine times more likely to develop delirium. ${ }^{9}$ A careful balance is therefore required as delirium is independently associated with poor functional recovery after hip fracture. ${ }^{10}$ Other agents such as non-steroidal anti-inflammatory drugs are effective but are associated with gastrointestinal and renal side effects, particularly in repeated doses. Intravenous paracetamol is a commonly used analgesic with a very good safety profile, and there is also some evidence to suggest that it is comparable to intravenous morphine in acute traumatic limb pain. ${ }^{11}$ However, ongoing pain relief with parenteral or enteral medication requires repeat administration, and in busy departments and wards it can be a challenge to provide timely and adequate pain relief.

In this study, there was no difference between the two groups in the use of intravenous morphine, intravenous or oral paracetamol, oral codeine and oral ibuprofen. There was, however, a wide range of analgesic strategies employed on the wards to treat hip fracture pain, suggesting there is scope to implement a structured approach to analgesia for these patients. 
Given our finding of equivalent pain relief, we can consider other factors when choosing a nerve block for this patient group. FIB is quick to deliver; $<5 \mathrm{~min}$ in one study, ${ }^{12}$ and half the time compared with 3 -in-1 by another. ${ }^{5}$ It suits the requirements of a modern ED with trainees who have not yet received ultrasound training. It is simple to anatomically define the correct landmarks and thus injection point, which is distant from the femoral neurovascular bundle. A correctly placed injection should have very little chance of intravascular injection or neural injury. FIB has a good safety profile, with only a clinically insignificant pneumoretroperitoneum, ${ }^{13}$ a temporary neuropathy ${ }^{14}$ and an accidental bladder ${ }^{15}$ puncture reported in literature. In a review of literature in 2007 , Brull et $a l^{16}$ reported the risk of neurological injury in femoral nerve blockade to be as low as 0.03 in one study. ${ }^{17}$

The least expensive way to deliver an effective block would be an anatomically guided 3 -in-1 block as it requires no additional equipment beyond a standard syringe and needle. The major expenditure associated with ultrasound is the machine itself. Although its usefulness would not be limited to nerve block delivery, this is a real consideration for EDs. The cost of a nerve-stimulator needle is in the region of $£ 3-5$, while a Tuohy needle costs around 50 pence. The drug costs between the two groups are similar with identical dosing. Rashid et $a l^{8}$ surveyed UK ED regional anaesthetic practice in hip fractures and reported that only $44 \%$ were routinely using nerve blocks for hip fractures. The most frequently cited reasons for not providing a nerve block were insufficient trained staff (36\%) and a lack of equipment $(22 \%)$. It was felt to be too time consuming by $10 \%$ of departments. Of those who did perform nerve blocks for hip fractures, $60 \%$ used a femoral nerve block and $22 \%$ FIB. Looking at all femoral fractures, a survey of $91 \%$ of UK departments reported that only 55\% regularly used femoral nerve blocks by any technique and only $10 \%$ regularly used ultrasound despite $74 \%$ having access to a machine. ${ }^{18}$ Clearly there is room to improve the uptake of regional techniques in managing hip fracture pain, and FIB would appear well suited to address this in ED.

\section{CONCLUSION}

FIB and 3-in-1 block are equivalent in reducing VAS pain scores in patients with a fractured neck of femur. Emergency physicians can have confidence in either technique when relieving pain in patients who present with a neck of femur fracture.

\section{Author affiliations \\ ${ }^{1}$ Academic Department of Emergency Care, Bristol Royal Infirmary, Bristol, UK ${ }^{2}$ Nottingham Clinical Trials Unit, University of Nottingham, Nottingham, UK ${ }^{3}$ Emergency Department, Derriford Hospital, Plymouth, UK \\ ${ }^{4}$ Academic Department of Military Emergency Medicine, Royal Centre for Defence Medicine (Research \& Academia), Birmingham, UK \\ ${ }^{5}$ Emergency Department, Wollongong Hospital, New South Wales, Australia \\ ${ }^{6}$ Faculty of Health and Life Sciences, University of the West of England, Bristol, UK}

Acknowledgements We are grateful to the staff in both participating emergency departments who recruited and randomised patients, and collected the data for this study.

Contributors I can confirm that the authors listed meet the International Committee of Medical Journal Editors Recommendations for the Conduct, Reporting, Editing and Publication of Scholarly Work in Medical Journals recommendations for authorship. PR: conception, design and analysis; drafting the article and revising it critically for important intellectual content; and final approval of the version published. AAM: design, analysis and interpretation of data; drafting the article or revising it critically for important intellectual content; and final approval of the version published. SB: design; revising it critically for important intellectual content and final approval of the version published. JE: design and acquisition of data; revising it critically for important intellectual content; and final approval of the version published. GE: acquisition of data; revising it critically for important intellectual content; and final approval of the version published. JES: acquisition of data or analysis and interpretation of data; drafting the article or revising it critically for important intellectual content; and final approval of the version published. JB: conception and design, analysis and interpretation of data; drafting the article or revising it critically for important intellectual content; and final approval of the version published.

Funding This research was funded by an unrestricted grant from the UK College of Emergency Medicine.

\section{Competing interests None.}

Ethics approval This study was approved by the Regional Ethics Committee, Frenchay Hospital, Bristol. The trial was registered with Current Controlled Trials (ISRCTN16152419) before patient recruitment commenced.

Provenance and peer review Not commissioned; externally peer reviewed.

Data sharing statement Anonymised individual patient data will be made available for secondary research, conditional on assurance from the secondary researcher that the proposed use of the data is compliant with the MRC Policy on Data Preservation and Sharing regarding scientific quality, ethical requirements and value for money. A minimum requirement with respect to scientific quality will be a publicly available prespecified protocol describing the purpose, methods and analysis of the secondary research, for example, a protocol for a Cochrane systematic review.

\section{REFERENCES}

1 National Institute for Health and Care Excellence. Clinical Guideline 124 Hip Fracture: the management of hip fracture in adults. London: NICE, 2011. http:/l guidance.nice.org.uk/CG124 (accessed 11/10/13).

2 College of Emergency Medicine. Clinical Standards for Emergency Departments. London: CEM, 2013. http://www.collemergencymed.ac.uk/Shop-Floor/Clinical\% 20Standards/default.asp (accessed 11-10-13).

3 Sharrock NE. Inadvertent ' 3 in 1 ' block following injection of the lateral cutaneous nerve of the thigh. Anesth Analg 1989:59:887-8.

4 Winnie AP, Ramamurthy S, Durrani Z. The inguinal paravascular technique of lumbar plexus anaesthesia: The "3-in-1" block. Anesth Analg 1973:52:989-96.

5 Newman B, McCarthy L, Thomas PW, et al. A comparison of pre-operative nerve stimulator guided femoral nerve block and fascia iliaca compartment block in patients with a femoral neck fracture. Anaesthesia 2013;69:899-903.

6 Taves DR. Minimization: a new method of assigning subjects to treatment and control groups. Clin Pharmacol Therapeut 1974;15:443-53.

7 Todd KH, Funk KG, Funk JP, et al. Clinical significance of reported changes in pain severity. Ann Emerg Med 1996;27:485-9.

8 Rashid A, Beswick E, Galitzine S. Regional analgesia in the emergency department for hip fractures: survey of current UK practice and its impact on services in a teaching hospital. Emerg Med J 2014;31:909-13.

9 Morrison R, Magaziner J, Gilbert M, et al. Relationship between pain and opiate analgesics on the development of delirium following hip fracture. J Gerontol A Biol Sci Med Sci 2003;58:76-81.

10 Marcantonio E, Flacker J, Michaels $M$, et al. Delirium is independently associated with poor functional recovery after hip fracture. J Am Geriatr Soc 2000;48:618-24.

11 Craig M, Jeavons R, Probert J, et al. Randomised comparison of intravenous paracetamol and intravenous morphine for acute traumatic limb pain in the emergency department. Emerg Med J 2012;29:37-9.

12 Monzon D, Iserson K, Vasquez J. Single fascia iliaca compartment block for post hip fracture pain relief. J Emerg Med 2007;32:257-62.

13 Shelley B, Haldane G. Pneumoretroperitoneum as a consequence of fascia iliaca block. Reg Anesth Pain Med 2006:31:582-3.

14 Atchabahian A, Brown A. Postoperative neuropathy following fascia iliaca compartment blockade. Anesthesiology 2001;94:534-6.

15 Blackford D, Westhoffen P. Accidental badder puncture: a complication of modified fascia iliaca block. Anaesth Intensive Care 2009;37:140-1.

16 Brull B, McCartney C, Chan V, et al. Neurological Complications After Regional Anaesthesia: Contemporary Estimates of Risk. Anesth Analg 2007;104:965-74.

17 Auroy $Y$, Benhamou D, Bargues L, et al. Major complications related to regional anaesthesia: the SOS regional anesthesia hotline service. Anesthesiology 2002:97:1274-80.

18 Mittal R, Vermani E. Femoral nerve blocks in fractures of femur: variation in the current UK practice and a review of the literature. Emerg Med J 2014;31:143-7. 